Rol del gerente educativo y el desempeño laboral en docentes de Educación Superior

Número Publicado el 05 de julio de 2018

DOI: $10.23857 /$ dc.v4i3.801

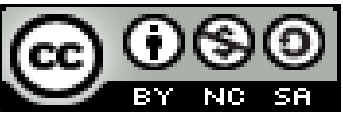

Ciencias de la educación

Artículo de investigación

\title{
Rol del gerente educativo y el desempeño laboral en docentes de Educación Superior
}

\section{Role of the educational manager and job performance in higher Education Teachers}

\section{Papel do gestor educacional e desempenho no trabalho em professores do ensino Superior}

\author{
Luzmila M. Valverde-Medina ${ }^{\mathrm{I}}$ \\ luzmilavalverde@yahoo.es \\ Leoaysa P. Ortiz-Delgado II \\ loayzaortiz@hotmail.com \\ Abraham T. Romero-Morales III \\ abrahanromero2014@hotmail.com
}

Recibido: 29 de enero de 2018 * Corregido: 20 de mayo de 2018 * Aceptado: 10 de junio de 2018

I. Profesor de Segunda Enseñanza Especialidad Químico-Biólogo, Licenciado en Ciencias de la Educación Especialidad Química y Biología, Diplomado Superior en Práctica Docente Universitaria, Especialista en Educación Superior, Magister en Investigación para el Desarrollo Educativo, Docente de la Universidad Luis Vargas Torres Esmeraldas, Ecuador.

II. Magister en Ciencias de la Educación Mención en Gestión Educativa y Desarrollo Social, Licenciada en Ciencias de la Educación, Especialidad Literatura y Castellano, Docente de la Universidad Técnica de Esmeraldas, Esmeraldas, Ecuador.

III. Magister en Docencia Mención Gestión en Desarrollo del Currículo, Licenciado en Ciencias de la Educación Mención Historia Y Geografía, Docente de la Universidad Técnica de Esmeraldas, Esmeraldas, Ecuador. 


\title{
Resumen
}

En el presente artículo se analizó la relación entre el rol de gerente educativo y el desempeño laboral de los docentes universitarios que laboran en el cantón Esmeraldas. La metodóloga se ubica dentro del marco de los estudios descriptivos - correlaciónales de campo, ya que tiene el propósito describir y medir el grado de relación que existe entre las dos variables objeto de estudio. La población o universo estuvo representada por 382 docentes universitarios del cantón de Esmeralda. El instrumento fue un cuestionario con una escala tipo Licker. Los datos aportados por el instrumento se procesaron estadísticamente a partir de un análisis descriptivo y chi cuadrado. Los resultados permitieron develar que el valor chi calculado con un valor de 0.000 es menor que el valor chi de la tabla con 0.05 , implicando que existe dependencia entre las variables, lo que permitió entre sus conclusiones que el desempeño laboral de los docentes universitarios está siendo afectado por el rol de mentor y facilitador que ejerce el gerente educativo universitario.

Palabras clave: rol de mentor; rol de facilitador; desempeño laboral y motivación.

\begin{abstract}
This article discussed the relationship between the role of educational Manager and the job performance of university teachers working in the Esmeraldas canton. The methodology is located within the framework of descriptive studies - correlative field, since it is intended to describe and measure the degree of relationship that exists between the two variables subject to study. The population or universe was represented by 382 University teachers from the canton of Emerald. The instrument was a questionnaire with a scale type Licker. Data provided by the instrument were statistically processed based on a descriptive analysis and chi square. The results allowed to reveal that the value calculated with a value of 0.000 chi is lower than the chi of the table with 0.05 , implying that there is dependence between the variables, which allowed among its conclusions that the job performance of teachers University is being affected by the role of mentor and facilitator that the University Educational Manager exercises.
\end{abstract}

Keywords: role of mentor; role of facilitator; work performance and motivation. 


\section{Resumo}

Neste artigo, analisou-se a relação entre o papel do gestor educacional e o desempenho no trabalho dos professores universitários que atuam no cantão de Esmeraldas. A metodologia está localizada no âmbito de estudos descritivos - campo de correlação, uma vez que tem por finalidade descrever e mensurar o grau de relacionamento existente entre as duas variáveis em estudo. A população ou universo foi representada por 382 professores universitários do cantão de Esmeralda. O instrumento foi um questionário com uma escala do tipo Licker. Os dados fornecidos pelo instrumento foram processados estatisticamente a partir de uma análise descritiva e qui-quadrado. Os resultados permitiram revelar que o valor chi com um valor de 0,000 é inferior a tabela de valores chi 0,05 , o que implica que não há dependência entre as variáveis, o que permitiu que as suas conclusões que o desempenho do trabalho dos professores universitários é ser afetado pelo papel de mentor e facilitador exercido pelo gerente de educação universitária.

Palavras chave: papel de mentor; papel do facilitador; desempenho no trabalho e motivação.

\section{Introducción}

La educación universitaria como organización es un proceso dinámico y complejo que ha de estar en constante mejoría y corresponderse a los diversos cambios que exige la sociedad, específicamente en lo que refiere a la actitud de sus docentes, para tal fin la misma demanda de una serie de acciones gerenciales relacionadas al rol que cumplen los gerentes educativos en este nivel al asumir un rol de facilitador y de viabilizador de la acción educativa, además de introducir en la institución: la motivación, el autoestima, la autenticidad, la satisfacción laboral, la empatía, la confianza, la comunicación, el diálogo y el control, entre otros; y así reaccionar de manera eficiente ante situaciones críticas para cumplir a cabalidad su rol de gerente dentro de la universidad.

Todo ello, en consonancia con los avances científicos y tecnológicos que se han venido operando en el mundo actual, en la que urgen docentes con capacidad de cambio, pero al mismo tiempo es necesario que el gerente educativo tome conciencia sobre la importancia del buen desempeño laboral docente que esté en sintonía con dichos cambios. A tal efecto, Schmelkes (2004), propone que la universidad ha de centrar su actividad en la satisfacción de las necesidades de sus beneficiarios, que son en primer lugar sus estudiantes, para lograrlo es necesario que quienes trabajan en las instituciones universitarias reconozcan la existencia de problemas y trabajen en equipo para descubrir y combatir 
sus causas. En este sentido, se propone el estudio de la relación entre el rol de gerente educativo y el desempeño laboral de los docentes universitarios que laboran en el cantón Esmeraldas.

\section{Desarrollo}

Ante la necesidad de cambios en los procesos y en las prácticas educativas universitarias, que consisten primordialmente en lograr que avancen hacia una calidad total se requiere de actitudes gerenciales que faciliten y promuevan un desempeño laboral direccionado al cumplimiento de las metas personales, profesionales e institucionales de la planta profesoral universitaria.

\section{Roles del gerente educativo}

En relación al de facilitador, el directivo debe fomentar el esfuerzo colectivo, lograr la unión y el trabajo en equipo y gestionar los conflictos interpersonales. Se debe orientar al proceso. Los comportamientos esperados incluyen intervenir ante las diferencias personales, aplicando técnicas de reducción del conflicto, desarrollar la unión y la moral, lograr el imput y la participación, y facilitar la resolución de problemas de grupos gestionando los conflictos interpersonales. Dentro de esta perspectiva, Torres (2011), afirma que el facilitador debe conocer la dinámica de los grupos, motivar y estimular constantemente la participación. Promover un ambiente de confianza y respeto. En otro orden de ideas, Harrington y Harrington (2007), afirman que es probable que el rol, más difícil en el ambiente de equipo sea el del facilitador. Hay muchos y diferentes criterios con respecto a lo que debe ser su función. Algunas instituciones utilizan al facilitador como un miembro total del equipo; otras creen que el facilitador debe ser un experto o tener gran conocimiento de los asuntos de equipos. En esencia se puede hablar de gerentes educativos que cumplen tres roles de facilitadores: el primero denominado integrador/coordinador, las tareas en este rol se asocian con la administración del proceso del trabajo en equipo de sus docentes y en comunicarse con los demás. El segundo rol el de especialista considerado el rol más tradicional y consiste en facilitar las reuniones de equipo con un enfoque en el proceso. Este facilitador no es un miembro de tiempo completo del grupo de trabajo y por último el rol de líder de sesiones es alguien que preside una reunión, a menudo en la forma de una sesión o taller de entrenamiento donde se requiera un facilitador tradicional.

\section{Desempeño Laboral}

Considerado como las acciones que deben ejecutar los individuos en función de los objetivos y metas de la organización en la cual laboran. En este sentido, Vroom (2011), señala que el desempeño laboral de las personas está en función de dos variables una representada por su capacitación intelectual y 
técnica y la otra por su motivación, para emplear sus capacidades en el cumplimiento de su labor. Por otra parte, Herzberg (2002), afirma que "el desempeño es una variable dependiente y está íntimamente relacionada con el nivel de satisfacción que experimenta el individuo con la jornada que realiza." (p.29). Por su parte, Smith (2011), afirma que en la teoría para rendir todo individuo sabe que su desempeño será evaluado, por sí mismo o por otras personas en función de alguna norma de calidad y que las consecuencias de su actuación determinará una evaluación favorable (éxito) o desfavorable (fracaso). En atención a esto, la teoría del desempeño gerencial define tres elementos esenciales. La expectativa, la motivación y el incentivo.

La expectativa. Se define como una anticipación cognoscitiva causada generalmente por algunos indicios en una situación que haría prever que una acción será guiada de una determinada consecuencia. Dessler (2009), sostiene que la teoría de las expectativas se refiere a que la motivación de un empleado aumenta cuando valora altamente un resultado particular y cuando siente una posibilidad razonablemente buena de alcanzar la meta deseada.

La Motivación. Está considerada como el impulso o energía que lleva al individuo a realizar una tarea. Chiavenato (2011), afirma que la motivación comienza cuando surge una necesidad, fuerza dinámica y persistente que origina el comportamiento. Cada vez que aparece una necesidad ésta rompe el estado de equilibrio del organismo y produce un estado de tensión, insatisfacción, inconformismo y desequilibrio que lleva al individuo a desarrollar un comportamiento o acción capaz de descargar la tensión y liberarlo de la inconformidad y del desequilibrio.

El Incentivo. Representa el atractivo que se ofrece en una situación y un motivo es una disposición a esforzarse por alcanzar cierta satisfacción y obtener algunos incentivos. Según Smith (2011) “ la finalidad del programa de incentivos es alentar a los funcionarios a que se esfuercen por hacer bien su trabajo y que se les reconozca y premie por sus logros.”. (p.98). En tal sentido, si se contribuye a alcanzar los objetivos de la organización, se reciben incentivos y en caso contrario sanciones.

\section{Metodología}

La metodóloga se ubica dentro del marco de los estudios descriptivos-correlacionales de campo, ya que tiene el propósito describir y medir el grado de relación que existe entre las dos variables objeto de estudio. Al respecto Chávez (2010), afirma que la investigación descriptiva es la que se orienta a redactar informes relacionados con el estado real de las personas, objeto situaciones o fenómenos tal cual se presentan en el momento de su recolección, describe lo que mide sin analizar inferencias ni 
verificar hipótesis y los estudios correlaciónales, según Sierra Bravo (2002), refiere que en estos estudios se establece la relación que existe entre dos o más variables, es decir se plantea la causaefecto de las variables en estudio. La población o universo estuvo representada por 382 docentes universitarios del cantón de Esmeralda. El instrumento fue un cuestionario con una escala tipo Licker. Los datos aportados por el instrumento se procesaron estadísticamente a partir de un análisis descriptivo y chi cuadrado.

\section{Resultados}

A continuación, se presenta el análisis de los datos que permitieron determinar la relación que existe entre el rol del gerente educativo y el desempeño laboral en docentes de educación superior.

Tabla 1. Rol de Mentor

\begin{tabular}{|c|c|c|c|c|}
\hline Sujetos & \multicolumn{2}{|c|}{ Docentes } & \multicolumn{2}{|c|}{ Directivos } \\
\hline Análisis / Alternativas & $\boldsymbol{F r}$ & $\%$ & $\boldsymbol{F r}$ & $\%$ \\
\hline SIEMPRE & 5 & 1.7 & 15 & 33.3 \\
\hline CASI SIEMPRE & 67 & 23.4 & 21 & 46.7 \\
\hline FRECUENTEMENTE & 179 & 62.6 & 7 & 15.6 \\
\hline ALGUNAS VECES & 24 & 8.4 & 2 & 4.4 \\
\hline NUNCA & 11 & 3.8 & 0 & 0 \\
\hline
\end{tabular}

Fuente: Elaboración Propia (2018)

En la tabla 1 , se indica que el $62.2 \%$ del total de los docentes investigados coincidieron en opinar que frecuentemente la gerencia educativa crea un clima de confianza en la organización, presiona a los subordinados para que sean eficientes, se muestra imparcial ante el personal que labora en la organización y evalúa el desempeño de los subordinados para aumentar el nivel de entusiasmo, mientras que el $46.7 \%$ del personal docente con cargo directivo señaló la alternativa casi siempre. Seguida del $23.4 \%$ de los docentes por horas que señalaron la alternativa casi siempre y el $33.3 \%$ del personal directivo que se inclinó por la alternativa siempre. Se destaca el hecho en la cual las alternativas siempre y nunca en opinión de los docentes alcanzaron los menores porcentajes de evaluación con el $1.7 \%$ y $3.8 \%$ respectivamente. 
Dom. Cien., ISSN: 2477-8818

Vol. 4, núm. 3, julio, 2018, pp. 177-188

Rol del gerente educativo y el desempeño laboral en docentes de Educación Superior

Tabla 2. Rol de Facilitador.

\begin{tabular}{|c|c|c|c|c|}
\hline \multirow{2}{*}{ Análisis / Alternativas } & \multicolumn{2}{|c|}{ Docentes } & \multicolumn{2}{|c|}{ Directivos } \\
\hline & $\boldsymbol{F r}$ & $\%$ & $\boldsymbol{F r}$ & $\%$ \\
\hline SIEMPRE & 5 & 1.7 & 10 & 22.2 \\
\hline CASI SIEMPRE & 20 & 7 & 27 & 60 \\
\hline FRECUENTEMENTE & 86 & 30.1 & 5 & 11.1 \\
\hline ALGUNAS VECES & 159 & 55.6 & 3 & 6.7 \\
\hline NUNCA & 16 & 5.6 & 0 & 0 \\
\hline
\end{tabular}

Fuente: Elaboración Propia (2018).

En la tabla.2, se indica que el 55.6\% del total de los docentes investigados coincidieron en opinar que algunas veces la gerencia educativa considera las necesidades del grupo como más importantes que las necesidades individuales, se preocupan por los problemas de los subordinados, buscan que los acuerdos sean explícitos y hacen que los conflictos se gestionen en forma constructiva y el $60 \%$ de los directivos se inclinaron por la alternativa casi siempre. Seguida del $30.1 \%$ de los docentes por horas que señaló la alternativa frecuentemente y el $22.2 \%$ de los directivos que se inclinó por la alternativa siempre. Se destaca el hecho en la cual las alternativas extremas de siempre y nunca en opinión de los docentes alcanzaron los menores porcentajes de evaluación con el 5.6\% y 1.7\% respectivamente.

Tabla 3. Expectativas del desempeño laboral.

\begin{tabular}{|c|c|c|c|c|}
\hline Sujetos & \multicolumn{2}{|c|}{ Docentes } & \multicolumn{2}{|c|}{ Directivos } \\
\hline Análisis / Alternativas & $\boldsymbol{F r}$ & $\%$ & $\boldsymbol{F r}$ & $\%$ \\
\hline SIEMPRE & 0 & 0 & 5 & 11.1 \\
\hline CASI SIEMPRE & 58 & 20.3 & 15 & 33.3 \\
\hline FRECUENTEMENTE & 76 & 26.6 & 21 & 46.7 \\
\hline ALGUNAS VECES & 129 & 45.1 & 4 & 8.9 \\
\hline NUNCA & 23 & 8 & 0 & 0 \\
\hline
\end{tabular}

Fuente: Elaboración Propia (2018) 
En la tabla 3, se indica que el 45,1\% del total de los docentes investigados coincidieron en opinar que algunas veces con su desempeño laboral se proponen a realizar las funciones inherentes a su cargo con esmero y dedicación, se sienten autorrealizados cuando realizan alguna actividad con excelencia y se proponen a responder oportunamente a todo cuanto le encomiendan dentro del proceso educativo, mientras que el $46.7 \%$ del total de los directivos indicó la alternativa frecuentemente. Seguida del $26.6 \%$ de los docentes que señaló la alternativa frecuentemente y el $33.3 \%$ de los directivos que se inclinó por la alternativa casi siempre. Se destaca el hecho en la cual la alternativa siempre en opinión de los docentes y la alternativa nunca en opinión de los directivos no presentan evaluación entre los investigados.

Tabla 4. Motivación del desempeño laboral

\begin{tabular}{lcccc}
\multicolumn{1}{c}{$\begin{array}{c}\text { Sujet } \\
\text { Análisis / Alternativas }\end{array}$} & $\boldsymbol{F r}$ & $\%$ & $\boldsymbol{F r}$ & $\boldsymbol{\%}$ \\
\hline SIEMPRE & 0 & 0 & 14 & 31.1 \\
\hline CASI SIEMPRE & 66 & 23.1 & 24 & 53.3 \\
\hline FRECUENTEMENTE & 161 & 56.3 & 7 & 15.6 \\
\hline ALGUNAS VECES & & & & \\
\hline NUNCA & 49 & 17.1 & 0 & 0 \\
\hline
\end{tabular}

Fuente: Elaboración Propia (2018)

En la tabla 4, se presentan los resultados del análisis frecuencial y porcentual del indicador motivación, en la que se indica que el $56.3 \%$ del total de los docentes investigados coincidieron en opinar que frecuentemente consideran que con su desempeño laboral el gerente promociona la motivación de su logro profesional, propician la motivación para realizar cualquier tipo de actividad dentro de la institución, reciben exaltación por el trabajo realizado y proporcionan medios para su perfeccionamiento, mientras que el $53.3 \%$ de los directivos consideraron la alternativa casi siempre. Seguida del 23,1\% de los docentes que señaló la alternativa casi siempre y el 31,1\% de los directivos que se inclinó por la alternativa siempre. Se destaca el hecho en la cual la alternativa siempre no presentó opinión entre los docentes por horas investigados. 
Tabla 5. Incentivos en el desempeño laboral

\begin{tabular}{lcccc}
$\begin{array}{c}\text { Sujetos } \\
\text { Análisis / Alternativas }\end{array}$ & $\boldsymbol{F r}$ & $\%$ & $\boldsymbol{F r}$ & $\%$ \\
\hline SIEMPRE & 0 & 0 & 23 & 51.1 \\
\hline CASI SIEMPRE & 159 & 55.6 & 19 & 42.2 \\
\hline FRECUENTEMENTE & & & & \multicolumn{2}{c}{$\begin{array}{c}\text { Docentes } \\
\text { Directivos }\end{array}$} \\
\hline ALGUNAS VECES & 98 & 34.3 & 3 & 6.7 \\
\hline NUNCA & 24 & 8.4 & 0 & 0 \\
\hline
\end{tabular}

Fuente: Elaboración Propia (2018)

En la tabla 5, se indica que el 55.6\% del total de los docentes investigados coincidieron en opinar que casi siempre reciben apoyo por su buen desempeño, igual sucedió con las oportunidades que reciben para resolver problemas dentro de la institución y para asumir responsabilidades, con esta misma frecuencia respondieron en relación al otorgamiento de medios para su desarrollo individual y un $51,1 \%$ del total de los directivos coincidieron en opinar que siempre brindaban incentivos. Seguida del $34,3 \%$ de los docentes que señalo la alternativa frecuentemente y el $42,2 \%$ de los directivos que se inclinó por la alternativa casi siempre. Se destaca el hecho en la cual la alternativa siempre entre los docentes no presentó opinión.

Tabla 6. Rol del Gerente Educativo

\begin{tabular}{|c|c|c|c|c|}
\hline \multirow{2}{*}{$\begin{array}{c}\text { Sujetos } \\
\text { Análisis / Alternativas }\end{array}$} & \multicolumn{2}{|c|}{ Docentes } & \multicolumn{2}{|c|}{ Directivos } \\
\hline & $\boldsymbol{F r}$ & $\%$ & $\boldsymbol{F r}$ & $\%$ \\
\hline SIEMPRE & 6 & 2.1 & 14 & 31.1 \\
\hline CASI SIEMPRE & 46 & 16.1 & 19 & 42.2 \\
\hline FRECUENTEMENTE & 125 & 43.7 & 9 & 20 \\
\hline ALGUNAS VECES & 104 & 36.4 & 3 & 6.7 \\
\hline NUNCA & 5 & 1.7 & 0 & 0 \\
\hline
\end{tabular}

Fuente: Elaboración Propia (2018) 
En la tabla 6, se presentan los resultados del análisis frecuencial de la dimensión rol del gerente educativo como producto del análisis conjunto de los indicadores rol del mentor y rol del facilitador, en la que se indica que el $43,7 \%$ del total de los docentes investigados opinaron que frecuentemente se cumplía con este rol, mientras que el $42.2 \%$ de los directivos señalaron la alternativa casi siempre. Seguida del $36.4 \%$ de los docentes que señaló la alternativa algunas veces y el $31.1 \%$ de los directivos que se inclinó por la alternativa siempre. Se destaca el hecho en la cual las alternativas extremas de siempre y nunca presentaron los menores porcentajes con sólo el 2,1\% y 1,7\% respectivamente en opinión de los docentes.

Tabla 7. Análisis del Desempeño Laboral

\begin{tabular}{|c|c|c|c|c|}
\hline \multirow{2}{*}{$\begin{array}{l}\text { Sujetos } \\
\text { Análisis / Alternativas }\end{array}$} & \multicolumn{2}{|c|}{ Docentes } & \multicolumn{2}{|c|}{$\begin{array}{l}\text { Directivos } \\
\text { Dirs }\end{array}$} \\
\hline & $F r$ & $\%$ & $\mathrm{Fr}$ & $\%$ \\
\hline SIEMPRE & 0 & 0 & 18 & 40 \\
\hline CASI SIEMPRE & 97 & 33.9 & 21 & 46.7 \\
\hline FRECUENTEMENTE & 123 & 43 & 6 & 13.3 \\
\hline ALGUNAS VECES & 58 & 20.3 & 0 & 0 \\
\hline NUNCA & 8 & 2.8 & 0 & 0 \\
\hline
\end{tabular}

Fuente: Elaboración Propia (2018)

En la tabla 7, se presentan los resultados del análisis frecuencial de la variable desempeño laboral como producto del análisis de la dimensión elementos del desempeño laboral a partir de los indicadores expectativas, motivación e incentivos, en la que se indica que el $43 \%$ del total de los docentes investigados opinaron para la alternativa frecuentemente se cumple, mientras que el 46,7\% del total de los directores indicó la alternativa casi siempre lo cumplen. Seguida del 33,9\% de los docentes que señaló la alternativa casi siempre y el $40 \%$ de los directivos que se inclinó por la alternativa siempre. Se destaca el hecho en la cual la alternativa que más favorece la evaluación de la variable no presenta opinión entre los docentes por ahora investigados. 
Rol del gerente educativo y el desempeño laboral en docentes de Educación Superior

Tabla 8. Relación Chi Cuadrado entre el desempeño laboral y el rol del gerente educativo.

\begin{tabular}{ll} 
Análisis / Posición & Valor \\
\hline VALOR P & 0.05 \\
\hline VALOR CHI & 0.000
\end{tabular}

Fuente: Elaboración propia (2018).

En la tabla 8, se presenta el análisis chi cuadrado entre las variables desempeño laboral y rol del gerente educativo, en la que se indica que el valor chi calculado con un valor de 0.000 es menor que el valor chi de la tabla con 0.05 , implicando que existe dependencia entre las variables, lo que evidencia que la variable desempeño laboral está siendo afectada por la variable rol del gerente educativo, lo que implica que ambas variables se interrelacionan afectando una a la otra.

\section{Conclusiones}

Los docentes investigados coincidieron en opinar que frecuentemente la gerencia educativa crea un clima de confianza en la organización, presiona a los subordinados para que sean eficientes, se muestra imparcial ante el personal que labora en la organización y evalúa el desempeño de los subordinados para aumentar el nivel de entusiasmo, mientras que los gerentes señalaron la alternativa casi siempre. Algunas veces en opinión de los docentes investigados la gerencia educativa considera las necesidades del grupo como más importantes que las necesidades individuales, se preocupan por los problemas de los subordinados, buscan que los acuerdos sean explícitos y hacen que los conflictos se gestionen en forma constructiva; mientras que los directivos se inclinaron por la alternativa casi siempre. Los docentes investigados coincidieron en opinar que algunas veces con su desempeño laboral se proponen a realizar las funciones inherentes a su cargo con esmero y dedicación, se sienten autorrealizados cuando realizan alguna actividad con excelencia y se proponen a responder oportunamente a todo cuanto le encomiendan dentro del proceso educativo, mientras que los directivos indicaron la alternativa frecuentemente.

Frecuentemente los docentes consideran que con su desempeño laboral el gerente promociona la motivación de su logro profesional, propician la motivación para realizar cualquier tipo de actividad dentro de la institución, reciben exaltación por el trabajo realizado y proporcionan medios para su 
Rol del gerente educativo y el desempeño laboral en docentes de Educación Superior

perfeccionamiento, mientras que los directivos consideraron la alternativa casi siempre. Casi siempre los docentes investigados reciben apoyo por su buen desempeño, igual sucedió con las oportunidades que reciben para resolver problemas dentro de la institución y para asumir responsabilidades, con esta misma frecuencia respondieron en relación al otorgamiento de medios para su desarrollo individual y los directivos coincidieron en opinar que siempre brindaban incentivos. En cuanto al rol que ejercen los gerentes se determinó que frecuentemente el gerente cumple con el rol de mentor y de facilitador, esto permite afirmar que en el nivel universitario existe un nivel medio de influencia en el rendimiento laboral de los docentes. Se estableció que frecuentemente durante el desempeño laboral no se cubren las expectativas del deber ser de los docentes del nivel universitario investigados, los resultados también evidencian poca motivación y ausencia de programas de incentivos que conlleven a la optimización del desempeño laboral de los docentes. El análisis chi cuadrado reveló que se establece una relación altamente significativa entre las variables desempeño laboral y rol del gerente educativo del nivel de educación superior, corroborando el nivel de dependencia entre ambas variables, esto permite afirmar que el desempeño laboral de los docentes universitarios está siendo afectado por el rol de mentor y facilitador que ejerce el gerente educativo.

\section{Referencias Bibliográficas}

Chávez, N. (2010). Introducción a la Investigación. Ars. Gráfica. Maracaibo Chiavenato, I. (2011). Administración de los Recursos Humanos. McGraw-Hill. Bogotá.

Dessler, G. (2009). Administración de Personal. IV Edición. Prentice-Hall. México.

Harrington, H. Y Harrington, J. (2007). Administración Total del Mejoramiento Continuo. La Nueva Generación. Editorial Mc. Graw Hill- México.

Herzberg, F. (2002). Una vez más como Motivar a sus Empleados. Editorial Mc. Graw Hill. México. Schmelkes, S. (2004). Hacia una mejor Calidad de nuestras Escuelas. Washington. OEA.

Sierra, R. (2002). Técnicas de Investigación Social. Teoría y ejercicios. Editorial Paraninfo.

Smith, C. (2011). Guías para Supervisores. Editorial Trillas. México.

Torres, M. (2011). La Praxis Andragógica. La Horizontalidad y la Participación en la Situación de Aprendizaje. Editado por el Consejo de Publicaciones de la Universidad de los Andes. MéridaVenezuela.

Vroom, V. (2011). Motivación y Alta Dirección. Editorial Trillas. México. 